\title{
Generation of fibroblasts overexpressing liver-specific PEPCK in a miniature pig model of human type 2 diabetes mellitus
}

\author{
YU-KYUNG KIM ${ }^{1 *}$, GEUN-SHIK LEE ${ }^{2 *}$, EUI-MAN JUNG ${ }^{3}$, \\ SANG-HWAN HYUN ${ }^{3}$, WOO-SUK HWANG ${ }^{1}$ and EUI-BAE JEUNG ${ }^{3}$
}

${ }^{1}$ SooAm Biotech Research Foundation, Seoul 137-851; ${ }^{2}$ Laboratory of Veterinary Physiology, College of Veterinary Medicine, Kangwon National University, Chuncheon, Gangwon 200-701; ${ }^{3}$ Laboratory of Veterinary Biochemistry and Molecular Biology, College of Veterinary Medicine, Chungbuk National University, Cheongju, Chungbuk 361-763, Republic of Korea

Received January 10, 2012; Accepted April 5, 2012

DOI: $10.3892 / \mathrm{mmr} .2012 .873$

\begin{abstract}
Type 2 diabetes mellitus (T2DM) is one of the most common complex metabolic disorders in humans, and is characterized by hyperglycemia and metabolic alterations. In T2DM, fasting hyperglycemia is attributed to excessive hepatic glucose production, and increased gluconeogenesis has been ascribed to increased transcriptional expression of phosphoenolpyruvate carboxykinase (PEPCK). In this study, we analyzed porcine PEPCK promoter activities to generate liver-specific expression vectors. We generated miniature pig fibroblasts overexpressing PEPCK via transgenes to provide an animal model of human T2DM. Various regions of the promoter showed high levels of activity in the presence of glucocorticoids, a PEPCK gene inducer, in liver cells compared to a positive control promoter. The selected promoter region for a liver-specific expression system was adopted based on the current targeting vector containing two selection markers, green fluorescence protein and a neomycin-resistance gene. The linearized vector was introduced into pig fibroblasts which facilitated liver-specific PEPCK overexpression and screening according to the two selection markers. In the present study, we used a liposome-mediated transfection protocol rather than a virus-mediated gene delivery system, since the virus may cause side effects. Following transfection, 46 colonies out of 33 transfection trials had positively integrated the overex-
\end{abstract}

Correspondence to: Dr Woo-Suk Hwang, SooAm Biotech Research Foundation, Bangbae 3 Dong, 1027-4 SooAm Building, Seoul 137-851, Republic of Korea

E-mail: hwangws@sooam.org

Dr Eui-Bae Jeung, Laboratory of Veterinary Biochemistry and Molecular Biology, College of Veterinary Medicine, Chungbuk National University, Cheongju, Chungbuk 361-763, Republic of Korea E-mail: ebjeung@chungbuk.ac.kr

*Contributed equally

Key words: phosphoenolpyruvate carboxykinase, diabetes mellitus, miniature pig, liver-specific expression pression vector, indicating that a relatively high transfection efficiency rate was obtained by the liposomal-mediated system. Thus, we recommend the optimal liver-specific expression system for safe and effective transfection of pig cells. We plan to use these cells for somatic cell nuclear transfer to produce piglets that overexpress liver-specific PEPCK as an animal model for human T2DM.

\section{Introduction}

Type 2 diabetes mellitus (T2DM), also known as non-insulindependent diabetes mellitus, is a metabolic disease characterized by elevated blood glucose concentrations that result from inadequate insulin action in insulin-sensitive tissues and from abnormal insulin secretion. T2DM is characterized by an imbalance of carbohydrate, fat and protein metabolism, primarily due to increased insulin resistance and relatively impaired insulin secretion (1). Tight control of blood glucose levels and the prevention of diabetic complications are the major goals of diabetes treatment (2). It is well-established that fasting hyperglycemia is a function of increased endogenous glucose production $(3,4)$. Several studies using a variety of methods have confirmed that increases in endogenous glucose production are due to increased gluconeogenesis as opposed to hepatic glycogenolysis (3,5-7).

Gluconeogenesis can be considered the reversal of glycolysis with the exception of a few specific steps in which unique enzymes are used either to bypass a thermodynamically unfavorable step or to avoid uncontrolled futile cycling (8). For example, the last step in glycolysis is the conversion of phosphoenolpyruvate (PEP) to pyruvate; this is a thermodynamically favorable reaction catalyzed by pyruvate kinase (PK). This process is accomplished in two enzymatic steps: conversion of pyruvate to oxaloacetate (OAA) catalyzed by pyruvate carboxylase (PC) followed by the conversion of OAA to PEP catalyzed by phosphoenolpyruvate carboxykinase (PEPCK) (9). The transcription of the PEPCK gene is heavily regulated and involves many transcriptional factors and other proteins (10-12). Accordingly, PEPCK is overexpressed in patients with all forms of diabetes in which gluconeogenesis is inappropriately high (13). A 7-fold overexpression of PEPCK in mice also results in hyperglycemia (14). PEPCK expression 
is known to be subjected to hormonal control through transcriptional regulation $(13,14)$. The PEPCK promoter contains multiple hormone-responsive elements, including ones specific for thyroid hormone, insulin, glucocorticoids and cAMP, suggesting that PEPCK is the only enzyme that controls the rate limiting step of gluconeogenesis in the liver (15).

Pigs have been extensively studied as a source of human organs and animal models for human disorders, since both humans and pigs share many physiological, anatomical and genetic similarities (16). In addition, successful somatic cell nuclear transfer (SCNT) technology has been established in pig models $(17,18)$. In the present study, we measured the promoter activities of the porcine PEPCK gene and generated a targeting vector containing the selected promoter region which facilitated PEPCK overexpression in pig liver. Additionally, we optimized a gene delivery system using liposome-mediated methods and screening systems using two well-known selection markers based on visual and antibiotic techniques.

\section{Materials and methods}

Cell culturing. Unless otherwise indicated, all cells were grown at $37^{\circ} \mathrm{C}$ and all cell culture materials were obtained from Invitrogen (Carlsbad, CA, USA). Porcine fibroblasts obtained from Yucatan pig fetus (Optifarm Solution Inc., Gyeonggi-do, Korea) on day 30 of pregnancy, and the H4IIE rat hepatoma cell line (ATCC, Manassas, VA, USA) were cultured in Dulbecco's modified Eagle's medium (DMEM) containing $10 \%$ fetal bovine serum (FBS), $50 \mathrm{U} / \mathrm{ml}$ penicillin and $50 \mu \mathrm{g} / \mathrm{ml}$ streptomycin.

Genomic DNA extraction and PCR. Genomic DNA of the porcine fibroblasts was isolated with a G-DEX ${ }^{\mathrm{TM}}$ IIc Genomic DNA Extraction kit (Intron Biotechnology, Suwon, Korea). Genomic DNA $(1 \mu \mathrm{g})$ was amplified in a $20 \mu \mathrm{l}$ PCR reaction containing 1 unit Ex-Taq polymerase (Takara, Otsu, Shiga, Japan), 2 mM dNTPs (Takara) and 10 pmol of each specific primer. All primers are provided in Table I. The PCR reactions were denatured at $95^{\circ} \mathrm{C}$ for $30 \mathrm{sec}$, annealed at $62^{\circ} \mathrm{C}$ for $30 \mathrm{sec}$ and extended at $72^{\circ} \mathrm{C}$ for 1 or $2 \mathrm{~min}$. The PCR products were subjected to cloning processes and/or separated on a $0.7 \%$ agarose gel, stained with ethidium bromide and photographed under UV illumination. The image was scanned using Gel Doc EQ (Bio-Rad, Hercules, CA, USA).

Vector construction. To measure promoter activity, various regions of the porcine PEPCK promoter [from -2,450, -1,836, $-1,086$ and -356 to +1 nucleotides $(\mathrm{nt}) ;+1=$ the transcriptional start site] were prepared by PCR using the genomic DNA. Amplified fragments were digested by $M l u \mathrm{I}$ and XhoI (Takara) and ligated into the promoter-less pGL3-Basic vector (Promega, Madison, WI, USA).

For the targeting vector, PEPCK cDNA was obtained by PCR with the specific primers of Table I from pig liver cDNA. The PCR product of PEPCK cDNA was digested with HindIII and $\mathrm{XbaI}$ (Takara), and then inserted into the pGL3 plasmid containing the PEPCK promoter (pPEPCK; $-1,836$ to $+1 \mathrm{nt}$ ) to replace the luciferase gene. Two selection markers, enhanced green fluorescence protein $(E G F P)$ and neomycin-resistance $(\mathrm{Neo})$ genes, were incorporated into the above plasmid. The
EGFP gene from pIRES2_EGFP (BD Biosciences, Franklin Lakes, NJ, USA) was isolated and ligated into the pIRES_Neo vector (BD Biosciences) at the EcoRV and BamHI sites using T4 ligase (Takara) according to the manufacturer's protocol. The two markers (EGFP and Neo) of the above plasmid were isolated and inserted into the PEPCK expression vector, pGL3-pPEPCK-PEPCK cDNA, at the SalI site. Finally, we generated the targeting vector, pGL3-PEPCK-PEPCK cDNApCMV-EGFP/Neo, and confirmed the whole sequences of the targeting vector by nucleotide sequence analysis (Genotech Co. Ltd., Daejeon, Korea).

Transient transfection and reporter gene assay. All of the transfection processes in this study were carried out using Lipofectamine $^{\mathrm{TM}} 2000$ (Invitrogen) according to the manufacturer's instructions. To control for different transfection efficiencies of the various PEPCK promoter-luciferase constructs, a Rous sarcoma virus (RSV)-lacZ plasmid was co-transfected. Briefly, H4IIE cells $\left(3 \times 10^{5}\right)$ were seeded in 6-well tissue culture plates 1 day before transfection. PEPCK promoter-luciferase construct $(4 \mu \mathrm{g})$ and $0.5 \mu \mathrm{g}$ of RSV-lacZ plasmid were co-transfected into the cells under serum-free DMEM. After incubating for $4 \mathrm{~h}$, the transfection medium was replaced with medium containing dexamethasone $(1 \mathrm{mM})$ and the cells were incubated for an additional $48 \mathrm{~h}$. A luciferase activity was performed using the Luciferase Assay System (Promega) and a GloMax 20/20 Luminometer (Promega). $\beta$-galactosidase activity was measured using a $\beta$-galactosidase Enzyme Assay System (Promega). The relative luciferase activity was calculated as the percentage $(\%)$ of relative luciferase/ $\beta$-galactosidase activity.

Establishment of transgenic fibroblast cell lines. The porcine fibroblasts were transfected with the targeting vector, pGL3-PEPCK-PEPCK cDNA-pCMV-EGFP/Neo, which was linearized by NotI (Takara), using Lipofectamine 2000 (Invitrogen). Twenty-four hours after transfection, the transfection medium was replaced with medium containing $250 \mu \mathrm{g} /$ $\mathrm{ml}$ of G418 (Roche, Basel, Switzerland) for 4 weeks. The presence of antibiotic-resistant colonies was visually confirmed using a fluorescence microscope (Nikon, Inc., Tokyo, Japan). After several passages, the positive cells were confirmed again the chromosomal incorporation of the targeting vector with PCR-based genotyping using the confirming primer sets shown in Table I.

Data analysis. Data are presented as the means \pm standard error of the mean (SEM). A statistical analysis was performed by Student's t-test, two-pair comparisons. $\mathrm{P}<0.05$ denoted statistical significance.

\section{Results}

Functional analysis of the porcine PEPCK promoter. To generate a tissue-specific targeting vector to overexpress PEPCK, we first analyzed the promoter activity of the porcine PEPCK gene. A series of porcine PEPCK promoters with sequential deletions were inserted into a well-known reporter plasmid, pGL3, containing the luciferase gene. The activities of the promoters were evaluated according to luciferase expression 
Table I. Primer sequences.

\begin{tabular}{lcll}
\hline Name & Restriction enzyme & Direction & Sequences (5' to 3') \\
\hline PEPCK promoter $(-2,457)$ & $M l u \mathrm{I}$ & Forward & GGG ACG CGT TGG GGG TGT TTT AAA GGG \\
PEPCK promoter $(-1,836)$ & $M l u \mathrm{I}$ & Forward & GGG ACG CGT TAA GGG CCC ACT GTT GTT A \\
PEPCK promoter $(-1,086)$ & $M l u \mathrm{I}$ & Forward & GGA CGC GTA GTC TGC TGC GCT GCT C \\
PEPCK promoter $(-356)$ & $M l u \mathrm{I}$ & Forward & GGA CGC GTA ACG GAC CGT GGC CAT \\
PEPCK promoter $(+1)$ & $X h o \mathrm{I}$ & Reverse & GGC TCG AGC ACC CTC GGG TAG ACG \\
PEPCK cDNA & HindIII & Forward & CCC AAG CTT ATG CCT CCT CAG CTC TCA AA \\
PEPCK cDNA & XbaI & Reverse & CCC TCT AGA GTC ACT CTG GCT GAT TCT CTG C \\
EGFP cDNA & EcoRV & Forward & GAT ATC CAC AAC CAT GGT GAG CAA GGG CGA \\
EGFP cDNA & BamHI & Reverse & GGA TCC TTA CTT GTA CAG CTC GTC CAT GCC \\
Confirming primer a & & Forward & GGA CGC GTA ACG GAC CGT GGC CAT \\
Confirming primer b & & Reverse & CGT TGG TGA AGA TGG TGT TG \\
Confirming primer c & & Forward & CAT GAA GCA GCA CGA CTT CT \\
Confirming primer d & & Reverse & CCT AGG AAT GCT CGT CAA GA
\end{tabular}
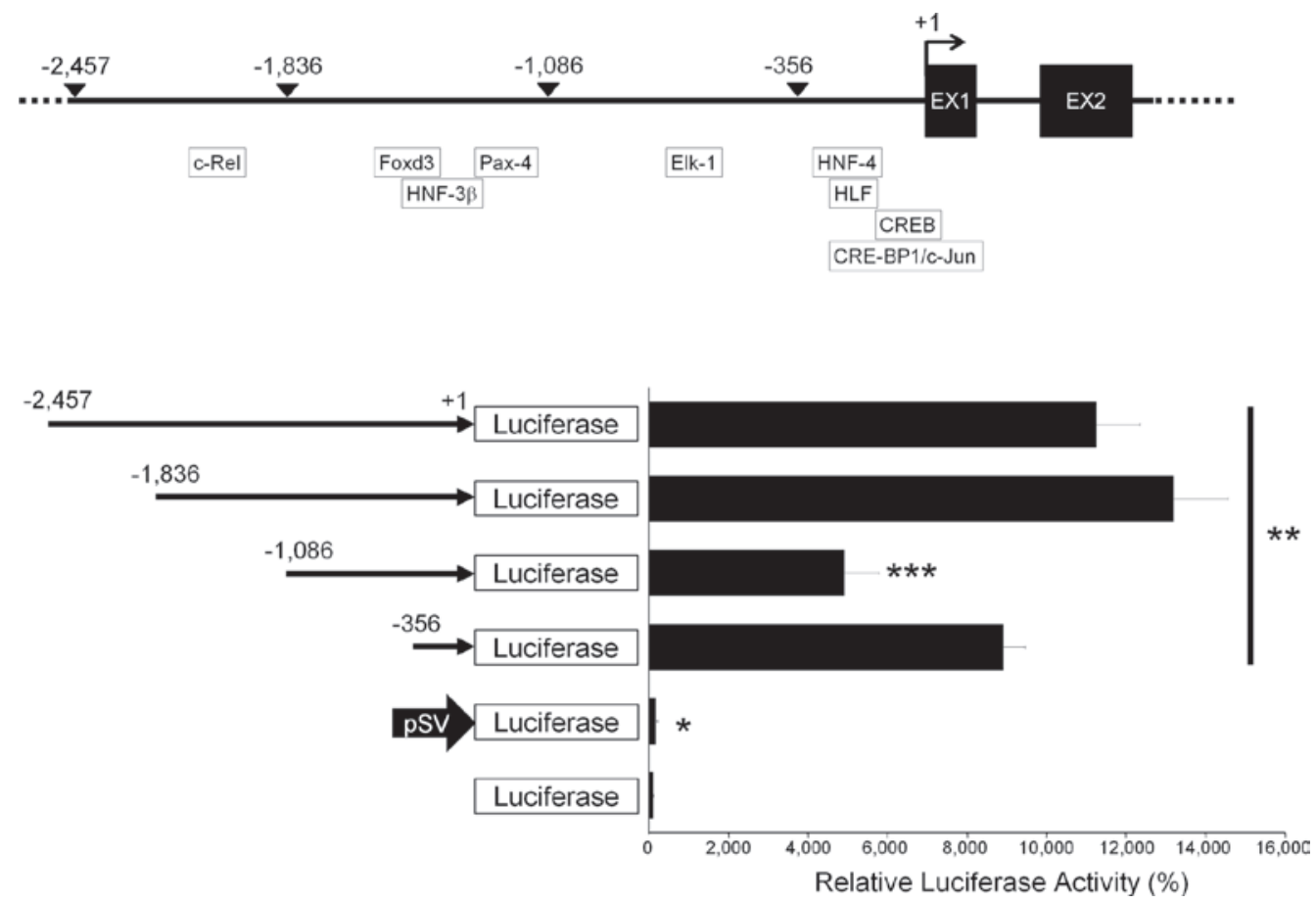

Figure 1. Analysis of pig PEPCK promoter activity in H4IIE cells. Schematic image depicting the porcine PEPCK promoter and putative transcriptional factors (upper panel). A set of deletion constructs containing the indicated regions of the PEPCK promoter was transiently transfected into H4IIE cells. The cells were treated for $24 \mathrm{~h}$ with $1 \mathrm{mM}$ dexamethasone to activate PEPCK gene transcription. An expression vector encoding RSV-lacZ was co-transfected to measure transfection efficiency. Promoter activity is represented as the percentage of induction after being normalized to $\beta$-galactosidase activity compared to cells transfected with luciferase activity under the control of an SV promoter (pSV) as positive control which was set at $100 \%$. Data represent the means \pm SEM of three independent experiments, each performed in triplicate. ${ }^{*} \mathrm{P}<0.05$ vs. promoter-less luciferase activity; ${ }^{* *} \mathrm{P}<0.05$ vs. luciferase activity under the control of the $\mathrm{SV}$ promoter; ${ }^{* * *} \mathrm{P}<0.05$ vs. the luciferase activity regulated by the promoter (from $-1,836$ to $+1 \mathrm{nt}$ ).

in the H4IIE hepatoma cell line after treatment with dexamethasone as a stimulator of PEPCK gene transcription (19). This system may mimic endogenous PEPCK gene expression.

As shown in Fig. 1, the SV promoter (pSV), a positive control, significantly induced luciferase expression when compared to the promoter-less luciferase gene alone. All of the PEPCK promoter variants produced $>40$-fold increases in promoter activities compared to the $\mathrm{pSV}$. The longer promoter regions $(-2,457$ to $+1 \mathrm{nt}$ or $-1,836$ to $+1 \mathrm{nt})$ resulted in a 130 - or
150 -fold increase in luciferase activity. The other two shorter promoter regions $(-1,086$ to $+1 \mathrm{nt}$ or -356 to $+1 \mathrm{nt})$ produced lower levels of activities than the two longer regions. These findings indicate that the regions between $-1,836$ and $-1,086 \mathrm{nt}$ may have certain enhancement elements.

Putative transcriptional factors were identified on the upper schematic diagram of the porcine PEPCK promoter region which was generated by MATCH ${ }^{\mathrm{TM}}$ software, public version 1.0 (BIOBASE GmbH, Wolfenbüttel, Germany). Although certain 


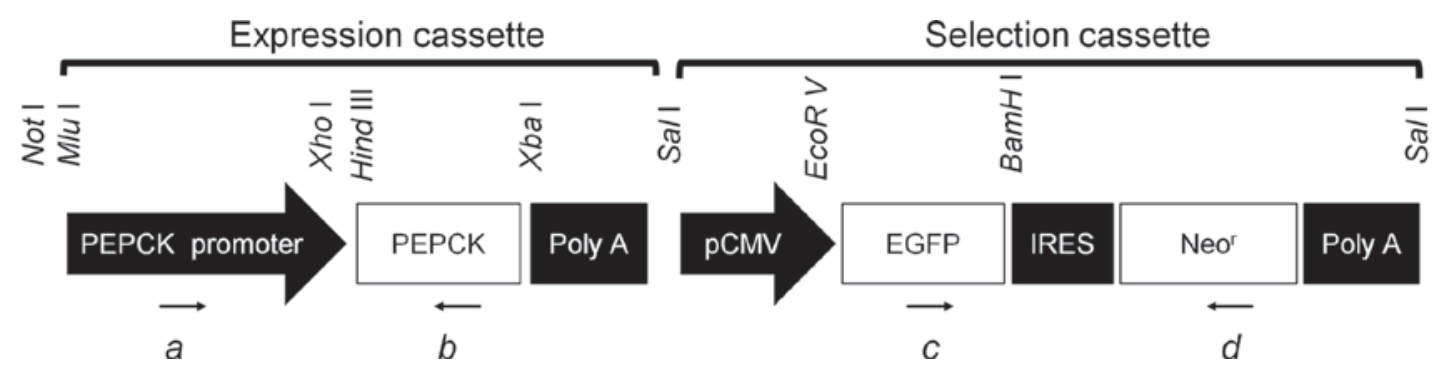

Figure 2. Schematic structure of the targeting vector. The targeting vector (pGL3-PEPCK promoter-PEPCK cDNA-pCMV-EGFP/Neo) contained two major cassettes: one for expression and the other for selection. The expression cassette facilitated liver-specific overexpression of the $P E P C K$ gene controlled by the porcine PEPCK promoter $(-1,836$ to $+1 \mathrm{nt})$. The selection cassette produces bi-cystic transcripts and enhances the expression of green fluorescence protein (EGFP) and neomycin-resistance (Neo) genes linked by an internal ribosomal entry site (IRES). Expression of the two marker genes was regulated by the CMV promoter (pCMV). Letters (a, b, c and d) and arrows indicate the locations and orientations of the PCR primers for Fig. 3C and D. The restriction endonuclease sites are also shown. Poly A, poly A tail signal sequences.

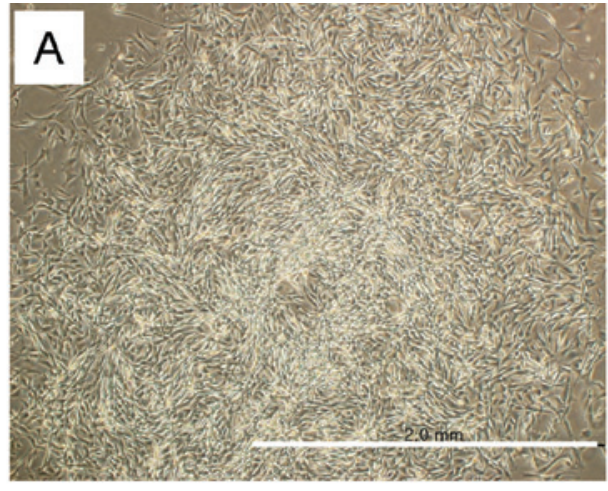

C

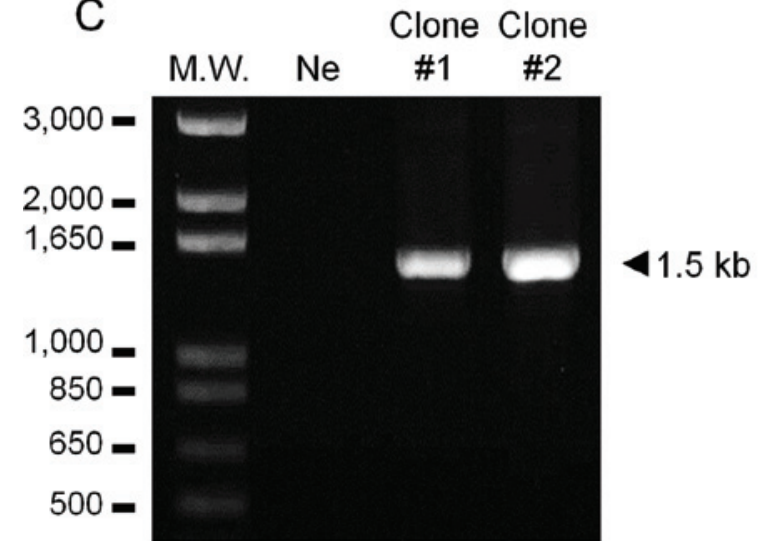

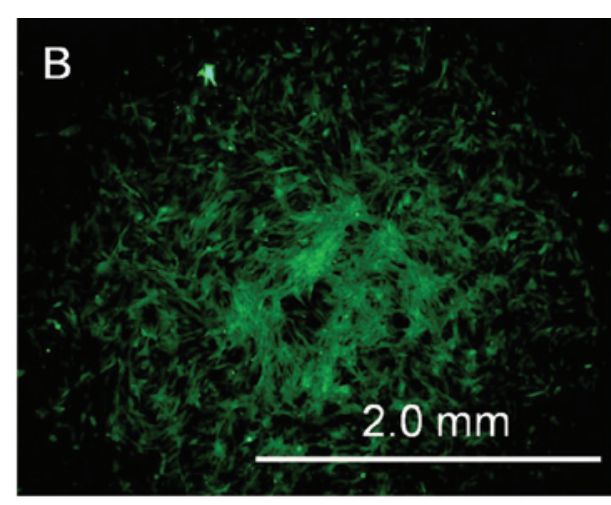

D
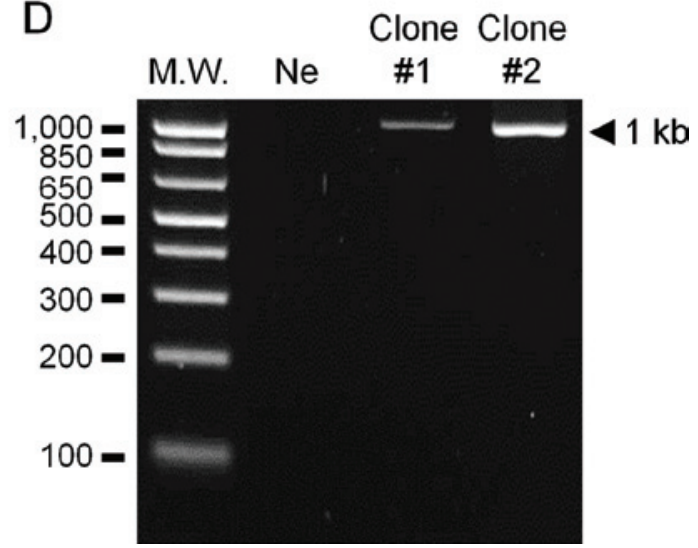

Figure 3. Visual and PCR-based confirmation of transgenic fibroblasts. Fetal fibroblasts from the Yucatan miniature pigs were transfected with the targeting vector, as described in Fig. 2, by Lipofectamine ${ }^{\mathrm{TM}} 2000$ and incubated with G418 for 4 weeks. (A) Antibiotic-resistant colony under light microscopy. (B) EGFP expression was observed with fluorescent microscopy. Genomic DNA was isolated from two representative positive colonies selected with antibiotics and visually confirmed GFP expression. PCR-based genotyping was then performed using the indicated primer set shown in Fig. 2. (C) Integration of the expression cassette of the targeting vector. (D) Insertion of the selection cassette of the vector. M.W., molecular weight (1 kb Ladder Plus); Ne, negative control (same PCR reactions without template).

activating factors (Foxd3, HNF-3 $\beta$ and Pax-4) for liver-specific transcription were located on the region between $-1,836$ and $-1,086 \mathrm{nt}$, we could not determine their role in the present study. However, our results showed that this region is important for liver-specific transcription. Thus, we selected the minimum region of the PEPCK promoter $(-1,836$ to $+1 \mathrm{nt})$ as a liverspecific regulator of the targeting vector.

Establishment of the targeting vector. The targeting vector consisted of two parts: an expression cassette and a selection cassette (Fig. 2). The expression cassette contained porcine
PEPCK cDNA controlled by its own promoter region $(-1,836$ to $+1 \mathrm{nt})$ which was previously confirmed by observing its maximum promoter activity in the liver cells. In addition, part of the selection cassette expressed bi-cystic transcripts, contained EGFP and Neo genes, and was regulated by a cytomegalic virus promoter (pCMV). These markers helped facilitate both antibiotic and visual screenings simultaneously during genetic modification.

Generation and confirmation of PEPCK overexpression in pig fibroblasts. The targeting vector was linearized and introduced 
Table II. Transfection efficiencies of the pig fibroblasts.
No. of transfection trials

No. of G418-resistant colonies

No. of PCR-positive colonies
No. of EGFP-positive colonies

into fibroblasts isolated from Yucatan pigs. The fibroblasts were maintained in medium containing neomycin $(250 \mu \mathrm{g} / \mathrm{ml}$ of G418) for 4 weeks and then screened for the presence of transgenic cells. The identity of neomycin-resistant colonies as transgenic fibroblasts was further confirmed by EGFP expression using a fluorescence microscope (Fig. 3A and B). Additionally, chromosomal integration of the targeting vector was identified by PCR-based analysis. As shown in Fig. 3C and $\mathrm{D}$, insertion of the expression and selection cassettes from the targeting vector was confirmed in two representative colonies. In total, we obtained 46 positive colonies after 33 rounds of pig fibroblast transfections using the liposomebased transfection system (Table II). These results indicate that the system we used was associated with a relatively high transgenic efficiency (88.5\%; 46 out of 52 colonies) although it produced a low number of positive colonies (1.6 colonies per transfection). Thus, our system involving dual screenings and liposome-mediated gene deliveries reduced the number of false-positive colonies during gene modification in pig fibroblasts. In the future, we will perform somatic cell nuclear transfer to produce piglets that overexpress liver-specific PEPCK as an animal model of human T2DM.

\section{Discussion}

The PEPCK promoter offers a good system for liver-specific gene expression. Previously, this promoter was examined to determine how genes respond to hormonal signals in a tissue-specific manner and the involvement of transcriptional elements in this process (19). The cAMP-mediated glucocorticoid responsiveness of the PEPCK promoter is robust in liver-derived cells, but weak in other cell types; this liver-specific responsiveness is mediated by a complex cAMP response element (CRE) in rodent models $(20,21)$. Consistent with the liver-specific nature of this response, several of the cis-elements required for maximal hormonal responsiveness bind to factors that are enriched in the liver (22).

Initially, we tested the activity of the pig PEPCK promoter in the H4IIE hepatic cell line. The PEPCK promoter (from -2,457 to $+1 \mathrm{nt}$ ) was subcloned into various regions and the activities of the constructs were evaluated. The shortest promoter region (from -356 to $+1 \mathrm{nt}$ ) was associated with high levels of promoter activities. This region contained well-characterized cis-elements from cAMP-responsive element binding protein (CREB), and CCAAT/enhancer-binding protein $\beta$ and c-Jun (CRE-BP/c-Jun). However, several transcriptional factors upstream of the PEPCK promoter were also involved in PEPCK gene expression, which has not been previously described. The regions between $-1,836$ and $-1,086 \mathrm{nt}$ contain other activating elements, while the region from $-1,086$ to -356 nt includes inhibiting regulating binding sites. Although we did not exten- sively characterize additional important upstream cis-elements in this study, our findings allow us to suggest that the promoter regions from $-1,836$ to +1 nt can be used to control exogenous gene overexpression in liver.

Antibiotic selection or visual markers have been commonly used when modifying genetic information of somatic cells for further SCNT-mediated cloning. In the present study, we simultaneously used two markers: green fluorescence protein and a neomycin-resistance gene. Antibiotic screening can be used to efficiently isolate transgenic cell colonies, while the visual marker provides a simple and effective means to identify transgenic piglets following SCNT. Although we used two selection markers, the fibroblasts may have a low fusion rate because of the extended culturing period and harsh environment due to the use of antibiotics and transfection reagents. To overcome these problems, we chose to use fetal cells from a Yucatan pig based on previous data. The use of fetal fibroblasts enabled us to achieve relatively high somatic cell cloning efficiencies in mammals $(23,24)$.

PEPCK is considered to be a key rate-controlling enzyme in the gluconeogenesis pathway (25). Normally, insulin rapidly and substantially inhibits $P E P C K$ gene transcription and gluconeogenesis in the liver and rat hepatoma cells (25). In genetically modified mice, inhibition of PEPCK by drug interventions results in hypoglycemia (26) and the ablation of the cytosolic PEPCK isoform results in non-viable offspring (27). On the other hand, excessive PEPCK expression in mice results in hyperglycemia (14), hyperinsulinemia and increased glucose turnover (26). Based on this evidence, we strongly suggest that overexpression of PEPCK in the liver may induce human T2DM-like pathogenesis in pigs.

Porcine models offer numerous advantages for studying human metabolic disorders, since pigs are monogastric omnivores like human, and have many anatomical and physiological similarities to humans (28). We can also perform many diagnostic and surgical techniques using pigs and directly transfer these to clinical practice. Through a long history of domestication, a wide variety of pig phenotypes is available, and this animal can be maintained under strict hygienic conditions, such as in specific pathogen-free and germ-free facilities. Additionally, the various phenotypes of pigs may be applicable to studying human diseases, such as obesity, diabetes and cardiovascular disease. These advantages led us to generate transgenic fibroblasts to replace the current rodent models of human diseases.

In conclusion, we successfully established a liver-specific gene controlling promoter system and developed miniature pig fibroblasts containing constructs for PEPCK overexpression. Further functional studies are required to characterize PEPCK overexpressing pig models. This stable cell line may serve as a valuable resource for SCNT. The piglets derived from these cells may also serve as a model for understanding the pathogenesis of human type 2 diabetes, and help develop novel diagnostic and treatment strategies.

\section{Acknowledgements}

This study was supported by a grant from the Next-Generation BioGreen 21 Program (no. PJ008323), Rural Development Administration, Republic of Korea. 


\section{References}

1. Holt RIG: Textbook of Diabetes. Wiley-Blackwell, Chichester West Sussex, 2010.

2. UK Prospective Diabetes Study (UKPDS) Group: Intensive blood-glucose control with sulphonylureas or insulin compared with conventional treatment and risk of complications in patients with type 2 diabetes (UKPDS 33). Lancet 352: 837-853, 1998.

3. Maggs DG, Buchanan TA, Burant CF, et al: Metabolic effects of troglitazone monotherapy in type 2 diabetes mellitus. A randomized, double-blind, placebo-controlled trial. Ann Intern Med 128: 176-185, 1998

4. Gastaldelli A, Miyazaki Y, Pettiti M, et al: Separate contribution of diabetes, total fat mass, and fat topography to glucose production, gluconeogenesis, and glycogenolysis. J Clin Endocrinol Metab 89: 3914-3921, 2004.

5. Magnusson I, Rothman DL, Katz LD, Shulman RG and Shulman GI: Increased rate of gluconeogenesis in type II diabetes mellitus. A $13 \mathrm{C}$ nuclear magnetic resonance study. J Clin Invest 90: 1323-1327, 1992.

6. Wajngot A, Chandramouli V, Schumann WC, et al: Quantitative contributions of gluconeogenesis to glucose production during fasting in type 2 diabetes mellitus. Metabolism 50: 47-52, 2001.

7. Kunert O, Stingl H, Rosian E, et al: Measurement of fractional whole-body gluconeogenesis in humans from blood samples using $2 \mathrm{H}$ nuclear magnetic resonance spectroscopy. Diabetes 52 2475-2482, 2003.

8. Pilkis SJ and Granner DK: Molecular physiology of the regulation of hepatic gluconeogenesis and glycolysis. Annu Rev Physiol 54: 885-909, 1992.

9. Hanson RW and Garber AJ: Phosphoenolpyruvate carboxykinase. I. Its role in gluconeogenesis. Am J Clin Nutr 25: 1010-1021, 1972.

10. Du K, Herzig S, Kulkarni RN and Montminy M: TRB3: a tribbles homolog that inhibits Akt/PKB activation by insulin in liver. Science 300: 1574-1577, 2003.

11. Jurado LA, Song S, Roesler WJ and Park EA: Conserved amino acids within CCAAT enhancer-binding proteins (C/EBP(alpha) and beta) regulate phosphoenolpyruvate carboxykinase (PEPCK) gene expression. J Biol Chem 277: 27606-27612, 2002.

12. Nakae J, Kitamura T, Silver DL and Accili D: The forkhead transcription factor Foxol (Fkhr) confers insulin sensitivity onto glucose-6-phosphatase expression. J Clin Invest 108: 1359-1367, 2001.

13. Veneziale CM, Donofrio JC and Nishimura H: The concentration of P-enolpyruvate carboxykinase protein in murine tissues in diabetes of chemical and genetic origin. J Biol Chem 258: 14257-14262, 1983.

14. Valera A, Pujol A, Pelegrin M and Bosch F: Transgenic mice overexpressing phosphoenolpyruvate carboxykinase develop non-insulin-dependent diabetes mellitus. Proc Natl Acad Sci USA 91: 9151-9154, 1994.
15. Short JM, Wynshaw-Boris A, Short HP and Hanson RW: Characterization of the phosphoenolpyruvate carboxykinase (GTP) promoter-regulatory region. II. Identification of cAMP and glucocorticoid regulatory domains. J Biol Chem 261: 9721-9726, 1986.

16. Gock H, Nottle M, Lew AM, d'Apice AJ and Cowan P: Genetic modification of pigs for solid organ xenotransplantation. Transplant Rev (Orlando) 25: 9-20, 2011.

17. Zhao J, Whyte J and Prather RS: Effect of epigenetic regulation during swine embryogenesis and on cloning by nuclear transfer. Cell Tissue Res 341: 13-21, 2010.

18. Dinnyes A, De Sousa P, King T and Wilmut I: Somatic cell nuclear transfer: recent progress and challenges. Cloning Stem Cells 4: 81-90, 2002.

19. Friedman JE: Role of glucocorticoids in activation of hepatic PEPCK gene transcription during exercise. Am J Physiol 266: E560-E566, 1994.

20. Roesler WJ, Simard J, Graham JG and McFie PJ: Characterization of the liver-specific component of the cAMP response unit in the phosphoenolpyruvate carboxykinase (GTP) gene promoter. J Biol Chem 269: 14276-14283, 1994.

21. Liu JS, Park EA, Gurney AL, Roesler WJ and Hanson RW: Cyclic AMP induction of phosphoenolpyruvate carboxykinase (GTP) gene transcription is mediated by multiple promoter elements. J Biol Chem 266: 19095-19102, 1991.

22. Roesler WJ, Vandenbark GR and Hanson RW: Identification of multiple protein binding domains in the promoter-regulatory region of the phosphoenolpyruvate carboxykinase (GTP) gene. J Biol Chem 264: 9657-9664, 1989.

23. Lee GS, Hyun SH, Kim HS, et al: Improvement of a porcine somatic cell nuclear transfer technique by optimizing donor cell and recipient oocyte preparations. Theriogenology 59: 1949-1957, 2003.

24. Yamazaki Y, Makino H, Hamaguchi-Hamada K, et al: Assessment of the developmental totipotency of neural cells in the cerebral cortex of mouse embryo by nuclear transfer. Proc Natl Acad Sci USA 98: 14022-14026, 2001.

25. Hanson RW and Reshef L: Regulation of phosphoenolpyruvate carboxykinase (GTP) gene expression. Annu Rev Biochem 66: 581-611, 1997.

26. Sun Y, Liu S, Ferguson S, et al: Phosphoenolpyruvate carboxykinase overexpression selectively attenuates insulin signaling and hepatic insulin sensitivity in transgenic mice. J Biol Chem 277: 23301-23307, 2002.

27. She P, Shiota M, Shelton KD, Chalkley R, Postic C and Magnuson MA: Phosphoenolpyruvate carboxykinase is necessary for the integration of hepatic energy metabolism. Mol Cell Biol 20: 6508-6517, 2000.

28. Petersen B, Carnwath JW and Niemann H: The perspectives for porcine-to-human xenografts. Comp Immunol Microbiol Infect Dis 32: 91-105, 2009. 